\title{
Relation between caval and pulmonary artery oxygen saturation in children
}

\author{
PER THAYSSEN, EBBE KLARHOLT \\ From the Department of Clinical Physiology, Odense University Hospital, Odense, Denmark
}

SUMMARY The flow relation between that in the superior vena cava and inferior vena cava was studied in order to estimate the oxygen saturation of mixed venous blood in connection with heart catheterisation of children between the ages of 5 and 8 years without cardiac shunts. The investigation includes a group of 19 children examined during halothane anaesthesia and a group of 40 examined under local anaesthesia. The groups were comparable in respect of age and body surface. The results show that the oxygen saturation of mixed venous blood (MVB) is higher under general anaesthesia than during local anaesthesia, and that the oxygen saturation in the former group is highest in the superior vena cava (SVC) and lowest in the inferior vena cava (IVC), while the reverse is the case during examination under local anaesthesia. Based on multiple regression analysis, it is advisable, for children between the ages of 5 and 8 years, to use the formula $M_{\text {VBalc }}=-0.85+0.81$ SVC +0.18 IVC for the estimation of the oxygen saturation in the mixed venous blood in children under general anaesthesia and the formula $\mathrm{MVB}_{\text {calc }}=10.21+0.52 \mathrm{SVC}+0.34$ IVC for children during local anaesthesia.

When estimating mixed venous blood in younger children and during general anaesthesia or both, more emphasis should be laid on the flow through the superior vena cava.

Calculation of the oxygen saturation of mixed venous blood is essential for the determination of the systemic cardiac output, when employing the Fick principle in patients with cardiac shunts.

The mixed venous blood depends on the flow and oxygen saturation in the superior vena cava, the inferior vena cava, and the coronary sinus. A total admixture of the blood streams from these three areas is first obtained in the pulmonary artery. ${ }^{1}$ No attention is paid to the influence of the flow from the coronary sinus during routine catheterisation, and for this reason the practical basis for the calculation of mixed venous blood normally only includes the flow in the two caval veins.

This basis for the calculation appears to differ in children subjected to examination under general anaesthesia from that in adults examined under local anaesthesia. In children between birth and 3 years without an intracardial shunt and examined under general anaesthesia, Thomsen ${ }^{2}$ found the best correlation between the mixed venous blood (MVB) measured directly (MVB meas) and MVB calculated (MVB ${ }_{\text {calc }}$ ) when using the formula:

Received for publication 8 October 1979

$$
\mathrm{MVB}_{\mathrm{calc}}=\frac{3 \mathrm{SVC}+\mathrm{IVC}}{4}
$$

where the superior vena cava (SVC) and inferior vena cava (IVC) are the oxygen saturation in the superior and inferior caval veins, respectively. Miller et $a l^{3}$ were unable to find a satisfactory formula for the calculation of mixed venous blood in children between birth and 10 years, examined under general anaesthesia.

The present investigation differs considerably in respect of both the type of anaesthesia and the age of the children. No previous report has included a reference group consisting entirely of children examined under local anaesthesia. In adults examined under local anaesthesia, the best correlation between $M_{\text {VBeas }}$ and $M_{\text {mB }}$ calc has been obtained using the formulae:

$$
\mathrm{MVB}_{\mathrm{calc}}=\frac{\mathrm{SVC}+\mathrm{IVC}}{2}
$$

(Nielsen and Fabricius) ${ }^{4}$ and 


$$
\mathrm{MVB}_{\mathrm{calc}}=\frac{3 \mathrm{SVC}+\mathrm{IVC}}{4}
$$

(Flamm et al. $\left.{ }^{5}\right)$.

The present retrospective study has been carried out in order to find the most suitable formula for the calculation of mixed venous blood in children.

\section{Patients and methods}

The material consists of 59 heart catheterisations of children in which the following criteria were complied with: (1) age between 60 and 96 months; (2) mixed venous blood between superior vena cava and inferior vena cava; and (3) no cardiac shunts.

Criterion (2) has been included partly in order to ensure that gross errors of method do not occur and partly to permit the estimation of the flow distribution between the superior and inferior caval veins.

Nineteen catheterisations were carried out under general anaesthesia (group A). All were performed under $\mathrm{N}_{2} \mathrm{O}-\mathrm{O}_{2}$-halothane anaesthesia, with the patient on spontaneous respiration. The oxygen content of the inspired air was between 33 and 50 per cent. The anaesthetics were supplemented with local infiltration of lignocaine 1 per cent in order to obtain light anaesthesia.

Forty catheterisations were carried out under local anaesthesia with lignocaine 1 per cent (group B). This was performed as an infiltration anaesthesia around the vein employed and only in five cases was it necessary to supplement this with intravenous pethidine.

The cardiac diagnoses in the two groups are shown in Table 1. The average age of the patients in group $A$ was 74 months and the average surface area $0.84 \mathrm{~m}^{2}$. In group $B$ the corresponding figures were 81 months and $0.88 \mathrm{~m}^{2}$.

The blood samples were withdrawn, as is the routine in the department, from the superior and inferior caval veins just before entering the right atrium (high IVC) and in the trunk of the pulmonary artery (MVB).

Table 1 Distribution of diagnoses among groups under general $(A)$ and local $(B)$ anaesthesia

\begin{tabular}{llc}
\hline Diagnosis & Group $A$ & Group B \\
\hline Normal & 5 & 24 \\
Aortic stenosis & 4 & 6 \\
Pulmonary stenosis & 3 & 7 \\
Operated heart disease & 4 & 2 \\
Aortic coarctation (mild) & 2 & 1 \\
Mitral regurgitation & 1 & - \\
\hline Total & 19 & 40 \\
\hline
\end{tabular}

The oxygen saturation determinations were carried out by reflection-photometry ("Oximeter"Elema-Schønander).

Calculation of the flow distribution between the superior and inferior caval veins was based on the following formula:

$$
\frac{\text { Q́svc }}{\text { Q'IVc }}=\frac{\text { MVB - IVC }}{\text { SVC }- \text { MVB }}
$$

where MVB, SVC, and IVC are the mean oxygen saturations in the trunk of the pulmonary artery, superior vena cava, and inferior vena cava, respectively.

Mixed venous blood was calculated using the following formulae:

(a) $\mathrm{MVB}_{\text {calc }}=\mathrm{SVC}$

(b) $\mathrm{MVB}_{\mathrm{calc}}=\frac{\mathrm{SVC}+\mathrm{IVC}}{2}$

(c) $\mathrm{MVB}_{\text {calc }}=\frac{3 \mathrm{SVC}+\mathrm{IVC}}{4}$

(d) $\mathrm{MVB}_{\text {calc }}=\frac{2 \mathrm{SVC}+\mathrm{IVC}}{3}$

(e) $\mathrm{MVB}_{\mathrm{calc}}=\frac{3 \mathrm{SVC}+2 \mathrm{IVC}}{5}$

(f) $\mathrm{MVB}_{\text {calc }}=-0.85+0.81 \mathrm{SVC}+0.18$ IVC

(g) $\mathrm{MVB}_{\text {calc }}=10.21+0.52 \mathrm{SVC}+0.34 \mathrm{IVC}$

Formulae (a), (b), and (c) have been previously used.

Formulae (d) and (e) have been worked out from the theoretical relation between the flows in the superior and inferior vena cavae in groups $A$ and $B$, respectively; an attempt has been made to use whole coefficient. Formulae (f) and (g) have been arrived at by multiple linear regression analysis of the measured values in groups $A$ and $B$, respectively. A comparison between $\mathrm{MVB}_{\text {meas }}$ and $M \mathrm{~B}_{\text {calc }}$ within the individual groups was carried out partly by means of linear regression analysis and partly using difference determinations. The regressions obtained in this manner were statistically analysed, with regard to a significant difference from the line of identity at a 5 per cent significance level. The mean values, mean differences, intercept, and slope have been compared using Student's $t$ test. 


\section{Results}

GROUP A

A significant correlation was found by means of linear regression analysis between mixed venous blood and superior vena cava $(r=0.90, p<0.001)$ and inferior vena cava $(r=0.57, \mathrm{p}<0.005)$, respectively, while no significant correlation could be found between mixed venous blood and age $(r=-0 \cdot 18)$ or surface area $(r=0 \cdot 12)$. Formula (f) was obtained by means of multiple regression analysis of the measured oxygen saturations in mixed venous blood, superior vena cava, and inferior vena cava, respectively.

Table 2 shows the mean values and scatter of mixed venous blood, superior vena cava, and inferior vena cava. The superior vena cava values are significantly higher than those of the inferior vena cava $(p<0.001)$. The relation between flows in the superior and inferior caval veins were calculated as $\mathbf{2 \cdot 0}$, using these mean values.

Table 2 Mean values $\pm 1 S D$ of oxygen saturation in mixed venous blood ( $M V B$ ), superior vena cava (SVC), and inferior vena cava (IVC)

\begin{tabular}{llll}
\hline & Group A & Group B & Probability \\
\hline MVB & $83.0 \pm 4.0$ & $75.9 \pm 3.5$ & $<0.001$ \\
SVC & $87.2 \pm 3.9$ & $73.8 \pm 4 \cdot 1$ & $<0.001$ \\
IVC & $\mathbf{7 4 . 4} \pm 6.6$ & $\mathbf{7 9 . 3} \pm 5.7$ & $<0.01$ \\
\hline
\end{tabular}

Table 3 shows the regression indices with the comparison of the measured and calculated values of mixed venous blood, when using formulae (a) to (f). In addition, the mean difference between the measured and calculated values is shown.

By inserting the values from group $B$ for superior vena cava and inferior vena cava into formula (f) a linear regression was found between $\mathrm{MVB}_{\text {calc }}$ and $M V B_{m e a s}$ which was significantly different from the lines of identity and also different from the linear regression obtained by using the measured values for SVC and IVC from group A in formula (f). The mean differences in both cases differed from zero $(p<0.001)$.

\section{GROUP B}

A significant correlation was found by means of linear regression analysis between mixed venous blood and superior vena cava $(r=0.75, p<0.001)$ and inferior vena cava $(r=0.72, p<0.001)$, respectively, while no significant correlation could be shown between mixed venous blood and age $(r=0 \cdot 15)$ or surface area $(r=0 \cdot 17)$. The previously mentioned formula (g) was derived from multiple regression analysis of the measured oxygen saturations in mixed venous blood, superior vena cava, and inferior vena cava.

The mean values and scatter of mixed venous blood, superior vena cava, and inferior vena cava are shown in Table 2. The values of the inferior vena cava are significantly higher than those of the superior vena cava $(p<0.001)$. The relation between flow in the superior and inferior vena cavae was calculated as 1.6 using these mean values of oxygen saturation.

Table 4 shows the regression indices with the comparison between measured and calculated values of mixed venous blood using formulae (a) to (e) and (g). In addition, the mean difference between the measured and calculated values is shown.

\section{Discussion}

The present investigation shows that the significantly highest values were obtained for mixed

Table 3 Formulae used with respective regression indices $\pm 1 S D$ in group $A$ (19 patients) ( $M V B_{\text {meas }}=a+b \times$ $M V B_{\text {calc) }}$

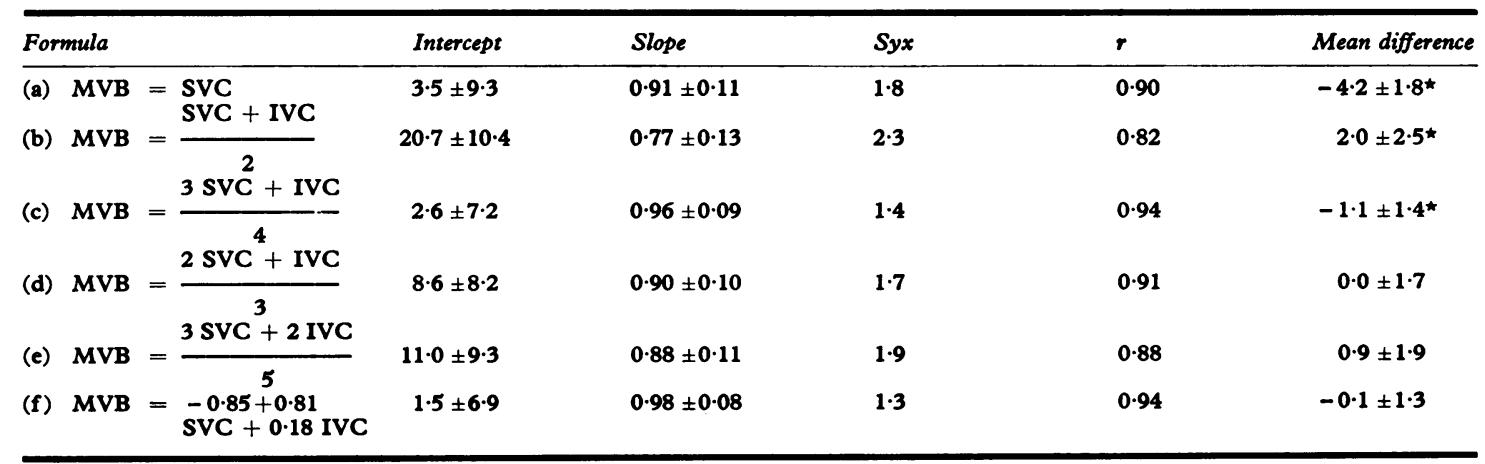

$\star p<0.05$ compared with line of identity. 
Table 4 Formulae used with respective regression indices $\pm 1 S D$ in group $B$ (40 patients) (MVBmeas $=a+b \times$ $M V B_{\text {calc) }}$

\begin{tabular}{|c|c|c|c|c|c|c|c|c|}
\hline \multicolumn{4}{|c|}{ Formula } & \multirow{2}{*}{$\frac{\text { Intercept }}{32 \cdot 5 \pm 6 \cdot 5^{\star}}$} & \multirow{2}{*}{$\frac{\text { Slope }}{0.59 \pm 0.09 \star}$} & \multirow{2}{*}{$\frac{S y x}{2 \cdot 4}$} & \multirow{2}{*}{$\frac{r}{0.73}$} & \multirow{2}{*}{$\frac{\text { Mean difference }}{1 \cdot 9 \pm 3 \cdot 0^{\star}}$} \\
\hline (a) & MVB & & $\begin{array}{l}\text { SVC } \\
\text { SVC + IVC }\end{array}$ & & & & & \\
\hline (b) & MVB & $=$ & $\overline{3 \mathrm{SVC}+\mathrm{IVC}}$ & $14 \cdot 0 \pm 4 \cdot 3^{\star}$ & $0.81 \pm 0.06^{\star}$ & $1 \cdot 4$ & 0.92 & $-0.8 \pm 1 \cdot 6^{\star}$ \\
\hline (c) & MVB & $=$ & $\overline{2 S V C C^{4}+I V C}$ & $10 \cdot 1 \pm 5 \cdot 3$ & $0.87 \pm 0.07$ & 1.6 & 0.90 & $0 \cdot 6 \pm 1 \cdot 6^{\star}$ \\
\hline (d) & MVB & $=$ & $\frac{3}{3 S V C+2 I V C}$ & $10 \cdot 2 \pm 4 \cdot 7^{\star}$ & $0.87 \pm 0.06^{\star}$ & $1 \cdot 4$ & 0.91 & $0.4 \pm 1.5$ \\
\hline (e) & MVB & $=$ & & $10 \cdot 4 \pm 4 \cdot 2^{\star}$ & $0.86 \pm 0.06^{\star}$ & $1 \cdot 3$ & 0.93 & $0 \cdot 0 \pm 1 \cdot 4$ \\
\hline (g) & MVB & $=$ & $\begin{array}{l}10.21+0.52 \\
\text { SVC }+0.34 \text { IVC }\end{array}$ & $2 \cdot 0 \pm 4 \cdot 7$ & $0.98 \pm 0.06$ & $1 \cdot 3$ & 0.93 & $0 \cdot 3 \pm 1 \cdot 3$ \\
\hline
\end{tabular}

$\star p<0.05$ compared with line of identity.

venous blood and superior vena cava, and the significantly lowest values for inferior vena cava under general anaesthesia. In addition, the inferior vena cava values are significantly higher than those of the superior vena cava under local anaesthesia, while the reverse is the case under general anaesthesia. The latter was also found by Thomsen ${ }^{2}$ in children between the ages of 0 and 3 years, examined under corresponding anaesthetic conditions. Thus, there appears to be an alteration in the circulation in children during light general anaesthesia in such a manner that the flow through the superior vena cava is increased and that through the inferior vena cava decreased. This is supported by animal experiments, ${ }^{6}$ which show a decreasing cerebral arteriovenous oxygen deficit in dogs under anaesthesia with rising concentrations of halothane and constant arterial $\mathrm{PCO}_{2}$. Further human investigations, ${ }^{78}$ have shown a reduced oxygen saturation in the venous blood from the splanchnic area during halothane anaesthesia. It is not possible from the present retrospective investigation to determine whether the change in the flow distribution results from an effect of halothane itself or a change in arterial $\mathrm{PCO}_{2}$, or both.

The relation between the superior and inferior vena cavae and mixed venous blood in a group consisting of children only, without cardiac shunts, under local anaesthesia, does not appear to have been the object of previous reports. We found that the values for the inferior vena cava were significantly higher than for the superior vena cava. The same finding has been made in adults. ${ }^{145}$

The following requirements were made in order to accept satisfactory estimations of mixed venous blood by one of the previously mentioned formulae (1) that $M V B_{\text {calc }}$ after comparison with $M V B_{\text {meas }}$ produced a linear regression with an intercept and a slope which did not differ significantly from 0 and 1, respectively; (2) a mean difference which did not differ significantly from 0 , and (3) a coefficient of correlation as near 1 as possible. The formulae which complied with these requirements were then mutually evaluated within the individual groups. We prefer the formulae with the greatest coefficient of correlation from those not mutually different.

If the coronary flow is ignored, we have found a flow relation between the superior and inferior caval veins in group $A$ of 2.0 . The formula (d) was arrived at from this value. The formulae were evaluated as described above, and in no case was the intercept found to be different from 0 or the slope different from 1. Formulae (a), (b), and (c) showed a mean difference different from 0 . No significant difference could be shown between the remaining formulae (d), (e), and (f). When compared with the formula recommended by Thomsen ${ }^{2}$ for the estimation of mixed venous blood in children examined under general anaesthesia and below the age of 3 years, we may conclude from the present investigation that one has to attribute less to the flow through the superior vena cava in older children. This may be one of the reasons why it was impossible for Miller et $a .^{3}{ }^{3}$ to find a satisfactory formula, as the patients in their material were between birth and 10 years of age. Other explanations might be the type of anaesthesia and intermittent positive pressure ventilation.

Using the criteria mentioned, we found only one formula in group B which did not differ from the ideal requirements, namely formula (g). The flow relation between the superior and inferior caval veins was 1.6 in this group, and it thus appears as though the flow in the superior vena cava must be considered higher in patients under general anaesthesia. The coefficients in formula $(\mathrm{g})$ can be 
seen to lie between the coefficients found by Nielsen and Fabricius, ${ }^{4}$ and Flamm et al., ${ }^{5}$ respectively, in adults.

As there is no difference between the patients in the two groups in respect of surface area, it may be concluded from the present investigation that it is advisable to employ different formulae for the estimation of mixed venous blood in children between the ages of 5 and 8 years, depending on whether the cardiac catheterisation is carried out under general or local anaesthesia.

\section{References}

${ }^{1}$ Barratt-Boyes BG, Wood EH. The oxygen saturation of blood in the venae cavae, right heart chambers, and pulmonary vessels of healthy subjects. $f \mathrm{Lab}$ Clin Med 1957; 50: 93-106.

'Thomsen A. Calculation of oxygen saturation of mixed venous blood in infants. Scand f Clin Lab Invest 1978; 38: 389-92.

${ }^{3}$ Miller HC, Brown DJ, Miller GAH. Comparison of formulae used to estimate oxygen saturation of mixed venous blood from caval samples. Br Heart $\mathcal{F} 1974$; 36: 446-51.

${ }^{4}$ Nielsen JS, Fabricius J. The blood flow in the caval veins at rest and during exercise in normal subjects. Acta Med Scand 1968; 183: 97-9.

${ }^{5}$ Flamm MMD, Cohn KE, Hancock EW. Measurement of systemic cardiac output at rest and exercise in patients with atrial septal defect. Am f Cardiol 1969; 23: 258-65.

${ }^{6}$ Smith AL. Dependence of cerebral venous oxygen tension on anesthetic depth. Anesthesiology 1973; 39: 291-8.

'Epstein RM, Deutsch S, Cooperman LH, Clement AJ, Price HL. Studies of the splanchnic circulation during halothane anesthesia in man (abstract). Anesthesiology $1965 ; 26$ : 246.

${ }^{8}$ Gattiker RI, Sessler AD, Lundborg RO, Swan HJC. Halothane and hepatic venous oxygen saturation (abstract). Anesthesiology 1965; 26: 246-7.

Requests for reprints to Dr Per Thayssen, Department of Clinical Physiology, Odense University Hospital, DK 5000 Odense C, Denmark. 